\title{
Simple Resonance Hierarchy for Surmounting Quantum Uncertainty
}

\author{
Richard L. Amoroso \\ Noetic Advanced Studies Institute, \\ 902 W 5400 N, Beryl, UT 84714 USA \\ amoroso@noeticadvancedstudies.us \\ $+7743020887$
}

\begin{abstract}
For a hundred years violation or surmounting the Quantum Uncertainty Principle has remained a Holy Grail of both theoretical and empirical physics. Utilizing an operationally completed form of Quantum Theory cast in a string theoretic Higher Dimensional (HD) form of Dirac covariant polarized vacuum with a complex Einstein energy dependent spacetime metric, $M_{4}: C_{4}$ with sufficient degrees of freedom to be causally free of the local quantum state, we present a simple empirical model for ontologically surmounting the phenomenology of uncertainty through a Sagnac Effect $R F$ pulsed Laser Oscillated Vacuum Energy Resonance hierarchy cast within an extended form of a Wheeler-Feynman-Cramer Transactional Calabi-Yau mirror symmetric spacetime backcloth.
\end{abstract}

\section{PHENOMENOLOGY VERSUS ONTOLOGY}

It is impossible by definition to violate the uncertainty principle, $\delta x \delta p_{x} \geq \hbar / 2$ or $\delta E \delta t_{x} \geq \hbar / 2$ within the framework of Copenhagen phenomenology arising from operation of a 'Heisenberg Microscope'. This is a fundamental empirical fact demonstrated by the Stern-Gerlach experiment where space quantization occurs for example along an arbitrary $z$ axis by continuous application of a non-uniform magnetic field to atomic spin structure [1], or by Young's double-slit experiment [2]. This scenario is cast in stone and is not the arena of our attack. The simplistic answer is 'do something else'! Our putative solution is contextual. Even though the solution is simple arriving at the new context is nontrivial and like any radically new idea will at first not be pleasing to many.

Recent advances in the new field of Interaction-Free Measurement (IFM) [3-5], a quantum mechanical interferometry procedure for detecting the state of an object without a phenomenological interaction occurring with the measuring device is a primitive precursor to our probability $\equiv 1$ model [6] utilizing additional degrees of freedom inherent in the supersymmetric regime of string/brane theory. Just as the UV catastrophe provided a clue for the immanent transition from Classical to Quantum Mechanics, duality in the Quantum Zeno Effect ${ }^{1}$ hints at another new horizon. IFM protocols have provided an intermediate indicia of this imanent scenario. The quantum Zeno paradox experimentally implemented in IFM protocols hints at the duality between the regular phenomenological quantum theory and a completed unitary or ontological model beyond the formalism of the standard Copenhagen interpretation. Utilizing extended theoretical elements associated with a new formulation for the topological transformation of a 'cosmological least unit', a putative empirical protocol for producing IFM with probability $\equiv 1$ is introduced in a manner representing a direct causal violation or absolute surmounting of the quantum Uncertainty Principle.

An interaction is any action, generally a force, mediated by an exchange particle like the photon in electromagnetic interactions. This physical concept of a fundamental interaction regards phenomenological properties of matter (Fermions) mediated by the exchange of an energy / momentum field (Bosons) as described by the Galilean, Lorentz or Poincairé groups of transformations. Here we introduce a new ontological type of homeomorphic transformation without an exchange particle mediated by an interactionless or energyless topological switching mechanism [7].

While considerations of the vacuum are of paramount concern for string theory, much of its putative essential parameters are ignored in the avid exploration of other details. The $P \equiv 1$ model relies heavily on the existence of a

\footnotetext{
1 Quantum states can be"frozen" by frequent measurement. Technically unitary time evolution can be suppressed by applying a series of strong fast pulses with proper symmetry decoupling a system from the decohering environment and thus preventing decoherence [6-9].
} 
Dirac polarized vacuum [8-10]. Of primary concern at this point of our development is its inclusion of extended electromagnetic theory [11-13] which is a key element in manipulating the structural-phenomenology of spacetime.

The experimental design, relyies on the utility of a new fundamental teleological action principle (synonymous with the unified field) inherent in the topological geometry of a covariant polarized Dirac vacuum putatively driving the evolution of self-organization in spacetime as an autopoietic complex system (reality itself), is developed to elucidate the methodology for surmounting uncertainty. The experimental apparatus, a multi-level interferometer, is designed to focus this noetic unitary field

As we shall see the protocol relies on the symmetry conditions of new self-organized cosmological parameters amenable to a resonant hierarchy of coherently controlled topological interactions able to undergo what Toffoli calls 'topological switching' [14] as the energyless basis for the Micromagnetics of information exchange. Finally to complete the concatenation we utilize theoretical concepts associated with the putative covariant polarized Dirac vacuum [8-10] forming a string theoretic spacetime background [15-17] also making correspondence between our ontological view of quantum theory and an extension of Cramer's Transactional Interpretation [18].
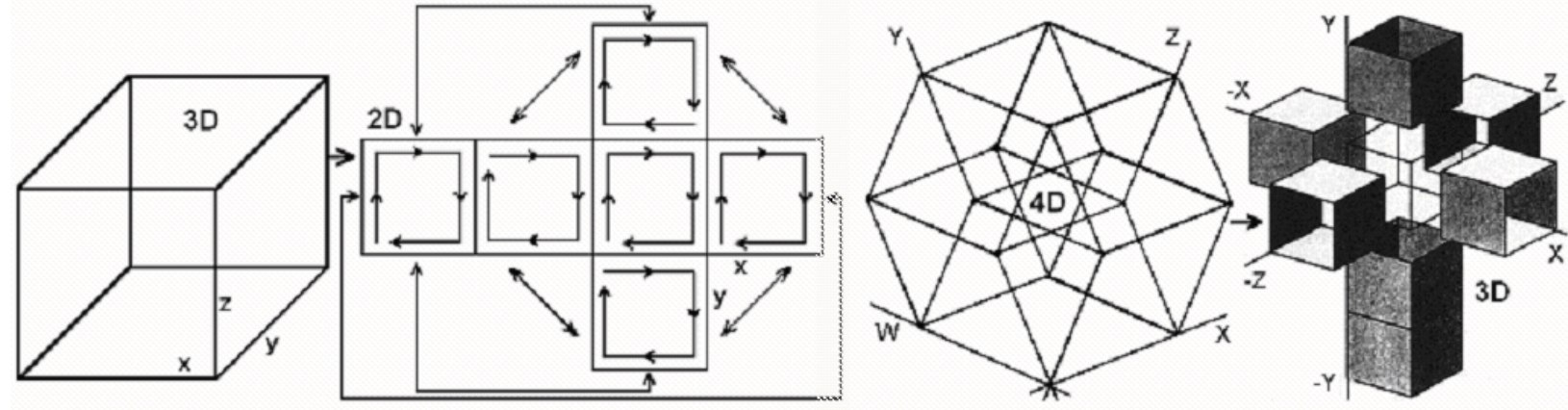

Figure 1 The suggestion is that the central translucent cube in the lower right represents a 'particle in a box' quantum state that through conformal scale-invariance remains physically real when the metaphor is carried to $12 \mathrm{D}$ where it becomes like the 'mirror image of a mirror image' and in that sense is causally free of the $E_{3}$ quantum state and thereby open to ontological information transfer in violation of Copenhagen uncertainty.

\section{COSMOLOGY IS KEY}

When physicists embraced the 3D Newtonian world view about a hundred years ago the universe was considered to be mechanical and predictable like a clockwork. Since the advent of QT reality is believed to be quantum and statistical or uncertain. Following this line of reasoning when a Theory of Everything (TOE) is realistically discovered based on a unitary field, should some form of monism be embraced? We postulate that cosmology is not uniquely based on any of these three conditions, but a continuous-state dynamic transformation of the three regimes comprised of a Wheeler-Feynman-Cramer complementarity [18-21] as outlined in Chap. 3. Physics has long resisted the role of the observer in physical theory; but in an anthropic cosmology the observer is an inherent key element or better said, the basis of observation [21]. This conundrum of the observer can be avoided here as its effects only become critical for process needing to control a much deeper region of spacetime.

Einstein stated that 'all of physics is based on measurements of duration and extension. Until now this has occurred within the parameters of a 4D Minkowski-Riemann spacetime metric under Gauge conditions utilizing various forms of the $E_{3} / \hat{M}_{4}$ Galilean-Lorentz-Poincairé transformations describing classical, quantum and relativistic conditions. These criteria are no longer sufficient and indeed our protocol for surmounting the uncertainty principle requires description of a new cosmological regime described by a new set of 12D transformations [22,23] we hope to call the Noetic Transformation because of its relevance to anthropic considerations. In this regard in spite of Bell's theorem, following Einstein's conundrum, we restate his complaint that quantum theory is incomplete and therefore wholly inadequate for some processes.

Cramer's transactional model of QT [18] has been ignored by most physicists for a variety of reasons we will not address here. This just means that when we bandy it about here as a key foundation of HAM cosmology it is foreign and not well understood. A Cramer transaction entails Wheeler-Feynman [19] future-past, standing-wave symmetry conditions which when extended to the HD SUSY regime readily lend themselves to mirror symmetry conditions inherent in our 12D version of M-Theory [24]. Further we suggest that the new 12D noetic transform adds additional piloting super-quantum potential [25] parameters, suggesting two forms, levels or regimes for quantum mechanics that of the observed $4 \mathrm{D}$ phenomenological interaction associated with the uncertainty principle; and the new HD ontological 'piloting' or anthropic guidance regime where reality itself is a transaction (see Fig. 2). Because the 
external world we observe is a limited subspace $[21,26]$ of a larger contiguous reality some elements are removed from perception by subtractive interferometry within the arrow of time.

In the standard Copenhagen Interpretation of QT an event emerges only as a result of measurement and objective reality is considered to be a probabilistic illusion. Cramer considers 'all off diagonal elements of the line element physically real' during the process of the offer-wave-confirmation-wave process preceding a transaction (event) [18]. We may call the final event a resultant of the conditions of Heisenberg Potentia. Here we still wish to consider reality illusory to the Minkowski observer.

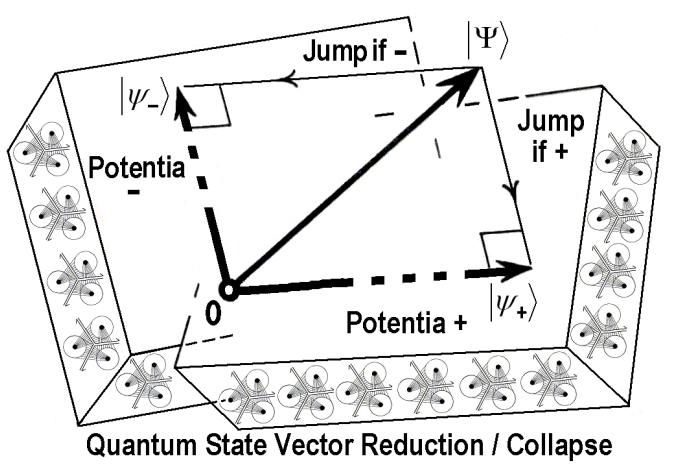

Figure 2 A way to look at a transaction as a collapse, $|\Psi\rangle$ to the 2D Euclidian plane from, in this case, an HD potentia of two possible orthogonal states, $\left|\psi_{+}\right\rangle,\left|\psi_{-}\right\rangle$.

Issues of the nature of the fundamental cosmological background continue to be debated with disparate views jockeying for philosophical supremacy; a scenario remaining tenable because experimental avenues for testing physics beyond the standard model have remained elusive. Here a putative empirical protocol is devised for manipulating the so-called covariant Dirac polarized vacuum (DPV) providing a methodology for both surmounting uncertainty and low energy protocols for testing string theory. The DPV has a sixty year history in the physics literature [8-10] which has for the most part been ignored by the main stream physics community for a number of philosophical conflicts. The problem of surmounting uncertainty is solved by the utility of additional degrees of freedom introduced by utilizing a multiverse cosmology and the associated extended theoretical elements.

\section{SPACETIME MICROMAGNETIC CONFORMATION TOPOLOGY}

An extensive body of literature exists for phenomena related to the zero-point field; but relative to noetic theory this work is considered metaphorically descriptive only of the 'fog over the ocean' rather than the structuralphenomenology of the ocean itself. Instead the deep structure of a real covariant Dirac polarized vacuum is utilized [8-10,28]. The Casimir, Zeeman, Aharanov-Bohm and Sagnac effects are considered evidence for a Dirac vacuum. New assumptions are made concerning the Dirac polarized vacuum relating to the topology of spacetime and the structure of matter cast in a 12D form of Relativistic Quantum Field Theory (RQFT) in the context of the new HAM cosmological paradigm [29-31]. In this anthropic cosmology the observed Euclidian-Minkowski spacetime present, $E_{3}-\hat{M}_{4}$ is a virtual standing wave of highly ordered Wheeler-Feynman-Cramer retarded-advanced future-past parameters respectively $[18,19]$. An essential ingredient of HAM cosmology is that a new action principle synonymous with the unified field arises naturally and is postulated to drive self-organization and evolution through all levels of scale [32-34].

In this context an experimental design [23,35] is introduced to isolate and utilize the new anthropic action to test empirically its putative ability to effect conformational structure of the topology of spacetime to surmount the usual phenomenologically based uncertainty in an ontological matter with probability $\equiv 1$.

Noetic Theory postulates that spacetime topology is 'continuously transformed' by the self-organizing properties of the long-range coherence [36,37] of the anthropic, élan vital or unitary noetic field [32,33,38-49]. In addition to manipulating conformational change, from the experimental results we attempt to calculate the energy Hamiltonian required to manipulate the Casimir topological conformation in terms of the noetic field equation, $F_{N}=E / R$ (unexpanded form. This resonant coupling produced by the teleological action of the anthropic noetic field driving its hierarchical self-organization has local, nonlocal and supralocal (complex HD) parameters [32]. The Schrödinger equation, extended by the addition of the de Broglie-Bohm quantum potential-pilot wave mechanism has been used to describe an electron moving on a neural manifold; but this is not a sufficient extension to describe anthropic 
noetic aspects of the continuous-state symmetry breaking of spacetime topology which requires further extension to include action of the noetic unitary field in additional dimensions.

The Noetic Field [32,33,38-51] produces periodic symmetry vari-ations with long-range coherence [35-37] that can lead to a critical Noetic Effect [32,39] of the Ising model lattice gas rotation of the Riemann sphere spacetime backcloth. This can be described by a form of double-cusp catastrophe dynamics (Fig. 9.9). Operationally the plane of equilibrium experiences sustained hyperincursion by the noetic field. The coupled modes of this process rely on a special form of the harmonic oscillator called the incursive oscillator [50-53]. There is a force of coherence [54]. For example for an Earth observer's temporal percep-tion, railroad tracks recede into a point at the horizon. For an atemporal eternal HD observer, the tracks remain parallel. This is the origin of the coherence force which forms a kind of logic gate driving equilibrium of the Casimir boundaries to parallel or degenerate modes thus giving rise to the possibility of effecting conformational states.

This is a boundary condition problem; here probably of the Born-von Karman type where the boundary conditions restrict the wave function to periodicity on a Bravais lattice of hexagonal symmetry, stated simply as $\psi\left(r+N_{i} a_{i}\right)=\psi_{r}$, where $i$ runs over the dimensions of the Bravais lattice, $a_{i}$ are the lattice vectors and $N_{i}$ are integers $[55,56]$. In this model presence of the periodic spherical rotation effects of the cyclical coherencedecoherence modes allow the action of the noetic field [32]. This Noetic Processing is governed by the fundamental equation of unitarity, $F_{N}=E / R$. Cyclotron resonance, logarithmic spiral, Kaluza-Klein hierarchy or genus-1 helicoid 'parking garage may maintain piloting by the noetic field or induce an electromotive 'radiation pressure' or topological switching coherence force, the Noetic Effect, on the topology of spacetime leading to conformational change in the static-dynamic [57-60] leapfrogging' of the Casimir boundary conditions of topological brane states.

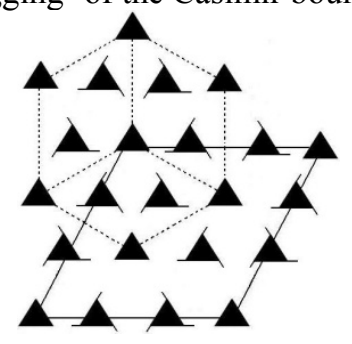

Figure $3 \mathrm{HD}$ emergence from a LD lattice gas. If the central vertex of the cube represents a Euclidian point, the 12 satellite points represent HD control parameters

We can't be sure yet which of the hierarchical formalisms might be the physical one until some empirical work is done. Intellectually we lean toward the concept of the action of a cyclotron resonance hierarchy acting on the genus-1 helicoid parking garage structure modulated by some form of Bessel function because this seems to meld well with catastrophe theory and the future-past symmetry breaking parameters we postulate in to be inherent in the structural-phenomenology of HAM continuous-state spacetime topology. The structural-phenomenology of atoms and molecules is full of domain walls amenable to description by combinations of Gauss' and Stokes' theorems ordered in terms of Bessel Functions where boundary conditions create resonant cavities built up by alternating static and dynamic Casimir conditions [57-60]. As frequency increases central peaks occur with opposite or zero polarity at the domain edges. These properties are relevant to Ising Model [61] spin flips of the domains of the Riemann-Block Spheres effecting homeostatic planes of equilibrium. The noetic effect can maintain equilibrium or produce catastrophes causing conformational change in the Casimir spacetime structures [62].

GEOMETRIC REPRESENTATION OF THE NOETIC FIELD EQUATION
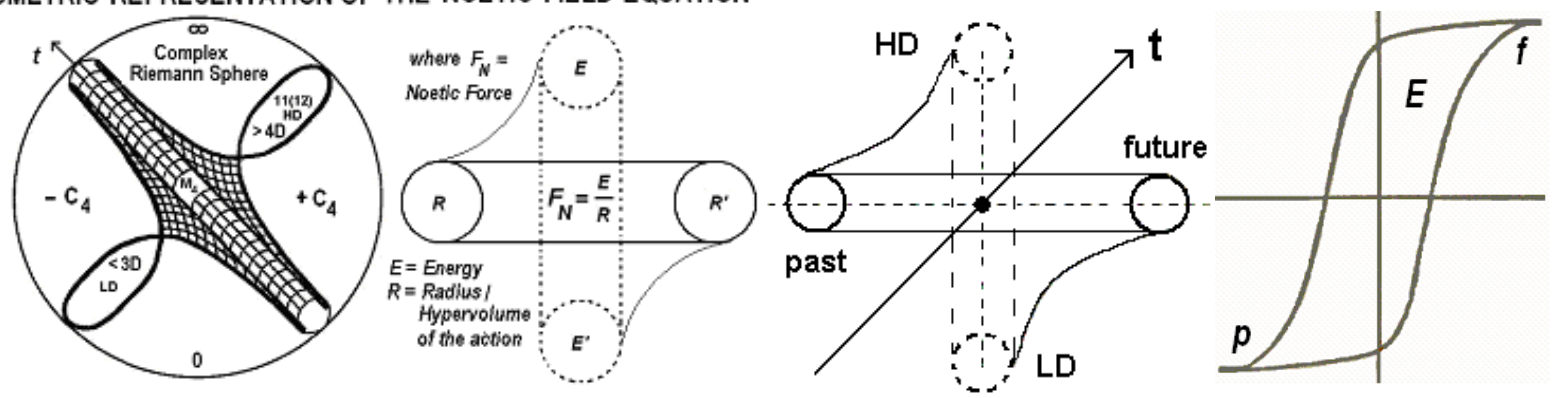

Figure 4 Topological and geometric idealizations of the noetic field equation describing an action of the noetic field, called the 'noetic effect', on a biological or spacetime manifold. 


\section{EXPERIMENTAL PROTOCOL}

Extrapolating Einstein's energy dependent or deformed spacetime metric, $\hat{M}_{4}$ [63-65] to a supersymmetric 12D standing-wave future-past advanced-retarded topology of the anthropic multiverse we have designed a spacetime resonance experiment for a covariant Dirac polarized vacuum which has properties like an 'ocean of light'. If this is true spacetime acts like a 'surface wave' on the upper regime of the complex self-organized Dirac Sea and is therefore amenable to descriptive methods of nonlinear dispersive wave phenomena generally of the basic form

$$
L(\mu)=\varepsilon N(\mu)
$$

where $L$ and $N$ are Linear and Nonlinear operators respectively in the linear limit where $\varepsilon=0$ with elementary dispersive wave solutions $\mu_{i}=A_{i} \cos \theta_{i}, \theta_{i}=k_{i} x-\omega\left(k_{i}\right) t$ for one dimension plus time where nonlinearity creates resonant interactions between the $\mu_{i}$ solutions and the Amplitude $A_{i}$ depends on $t$, creating potentially substantial effects where initial absent modes can become cumulative interactions producing shock wave effects.

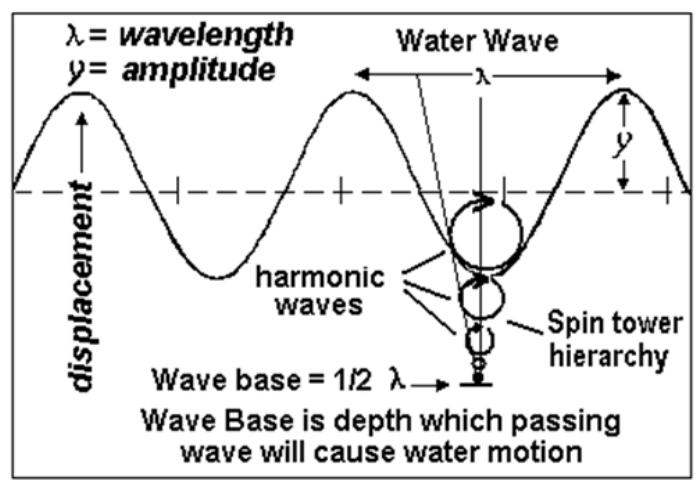

SPACETIME RESONANCE HAS SPHERICAL SYMMETRY
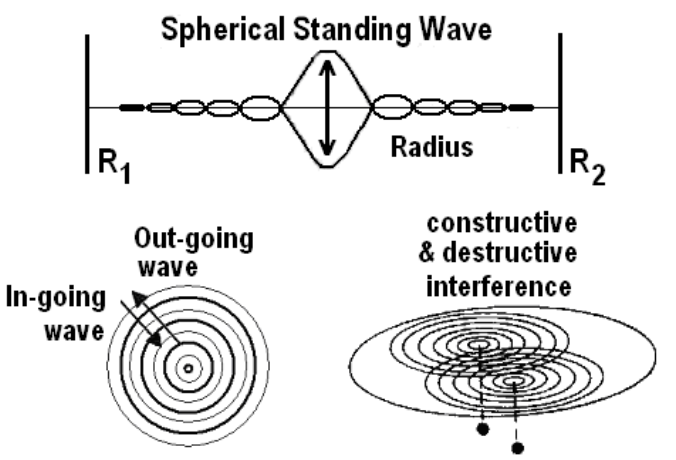

Figure 5 A) The spacetime topological hierarchy may have properties like water waves where the wave moves but the water remains stationary. B) The Dirac polarized vacuum has hyperspherical symmetry. a) Metaphor for standing-wave present showing future-past elements, $R_{1}, R_{2}$, eleven of twelve dimensions suppressed for simplicity. b) Top view of a) a 2D spherical standing-wave. c) Manipulating the relative phase of oscillations creates nodes of destructive and constructive interference.

Motion of a one dimensional classical harmonic oscillator is given by $q=A \sin (\omega t+\varphi)$ and $p=m \omega A \cos (\omega t+\varphi)$ where $A$ is the amplitude and $\varphi$ is the phase constant for fixed energy $E=m \omega^{2} A^{2} / 2$. For state $|n\rangle$, with $n=0,1,2 \ldots \infty$ and Hamiltonian $E_{n}=(n+1 / 2) \hbar \omega$ the quantum harmonic oscillator becomes

$$
\left\langle n\left|q^{2}\right| n\right\rangle=\hbar / 2 m \omega\left\langle n\left|\left(a^{\dagger} a+a a^{\dagger}\right)\right| n\right\rangle=E_{n} / m \omega^{2}
$$

and

$$
\left\langle n\left|p^{2}\right| n\right\rangle=1 / 2(m \hbar \omega)\langle n| a^{\dagger} a+a a^{\dagger}=m E_{n}
$$

where $a \& a^{\dagger}$ are the annihilation and creation operators, $q=\sqrt{\hbar / 2 m \omega}\left(a^{\dagger}+a\right)$ and $p=i \sqrt{m \hbar \omega / 2}\left(a^{\dagger} a\right)$. For the $3 \mathrm{D}$ harmonic oscillator each equation is the same with energies $E_{x}=\left(n_{x}+1 / 2\right) \hbar \omega_{x}$, $E_{y}=\left(n_{y}+1 / 2\right) \hbar \omega_{y}$ and

$$
E_{z}=\left(n_{z}+1 / 2\right) \hbar \omega_{z}[66,67] .
$$

In Dubois' notation the classical 1D harmonic oscillator for Newton's second law in coordinates $t$ and $x(t)$ for a mass $m$ in a potential $U(x)=1 / 2\left(k x^{2}\right)$ takes the differential form

$$
\frac{d^{2} x}{d t^{2}}+\omega^{2} x=0 \quad \text { where } \quad \omega=\sqrt{k / m}
$$

which can be separated into the coupled equations [68-71] 


$$
\frac{d x(t)}{d t}-v(t)=0 \quad \text { and } \quad \frac{d v(t)}{d t}+\omega^{2} x=0 .
$$

From incursive discretization, Dubois creates two solutions $x(t+\Delta t) v(t+\Delta t)$ providing a structural bifurcation of the system which together produce Hyperincursion. The effect of increasing the time interval discretizes the trajectory. This represents a background independent discretization of spacetime [50-53].
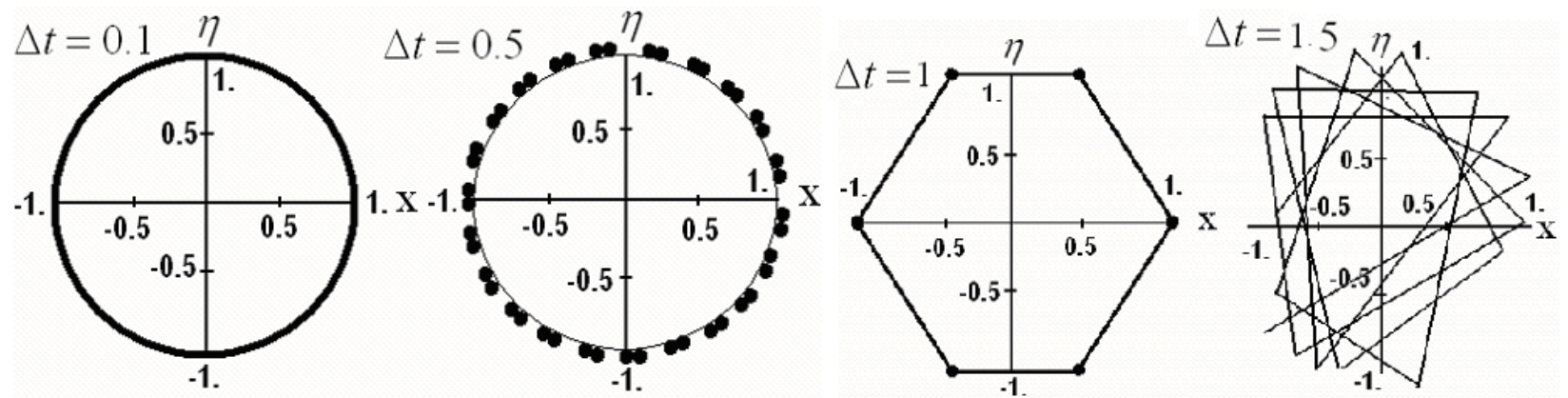

Figure 6 Numerical simulation of the phase space trajectory of the Dubois superposed incursive oscillator based on coordinates and velocities $x_{n}=1 / 2\left[x_{n}(1)+x_{n}(2)\right] v_{n}=1 / 2\left[v_{n}(1)+v_{n}(2)\right]$ is shown in the figure for values of $\Delta \tau=\omega t$ equal to $0.1,0.5,1.0$ and 1.5. Initial conditions are $\chi_{0}=1, \eta_{0}=0 \& \tau_{0}=0$ with total simulation time $\tau=\omega t=8 \pi$. Figure adapted from [50-53].

\section{INTRODUCTION TO P $\equiv 1$ EXPERIMENTS}

In a homogeneous magnetic field, the forces exerted on opposite ends of the dipole cancel each other out and the trajectory of the particle is unaffected. if the particles are classical "spinning" particles then the distribution of their spin angular momentum vectors is taken to be truly random and each particle would be deflected up or down by a different amount producing an even distribution on the screen of a detector. instead, quantum mechanically, the particles passing through the device are deflected either up or down by a specific amount. this means that spin angular momentum is quantized (also called space quantization), i.e. it can only take on discrete values. there is not a continuous distribution of possible angular momenta. this is the usual fundamental basis of the standard quantum theory and where we must introduce a new experimental protocol to surmount it. This is the crux of our new methodology: If application of a homogeneous magnetic field produces quantum uncertainty upon measurement, then "do something else".

In NMR spectroscopy often it is easier to make a first order calculation for a resonant state and then vary the frequency until resonance is achieved. Among the variety of possible approaches that might work best for a specific quantum system, if we choose NMR for the Noetic Interferometer it is relatively straight forward to determine the spin-spin resonant couplings between the modulated electrons and the nucleons; but achieving a critical resonant coupling with the wave properties of matter and the spacetime backcloth is another matter. Firstly, for HAM cosmology $\hbar$ is not a rigid barrier as in Standard Model Big Bang-Copenhagen cosmology; $\hbar$ is a virtual limit of past-advanced elements of the continuous-state standing-wave present as it cyclically recedes into the past where the least unit [72] cavities tiling the spacetime backcloth can have radii $\leq$ the Larmour radius of the hydrogen atom $[32,33,73,74]$. This new Planck length oscillates through a limit cycle from the Larmour radius of the hydrogen atom to standard $\hbar$. This is like a wave-particle duality - Larmour radius at the future-retarded moment and $\hbar$ at the past-advanced moment. The dynamics are different for future-retarded elements which have been theorized to have the possibility of infinite radius for $\mathrm{D}>4$ [26]. This scenario is a postulate of string theory. Considering the domain walls of the least-unit structure, the $\hbar$-Larmour regime is considered internal-nonlocal and the Larmour-infinity regime considered external-supralocal [29-31].

For simplicity we introduce our review of NMR concepts for the hydrogen atom, a single proton with magnetic moment $\mu$, angular momentum $J$ related by the vector $\mu=\gamma J$ where $\gamma$ is the gyromagnetic ratio and $J=\hbar I$ where $I$ is the nuclear spin. The magnetic energy $U=-\mu \cdot B$ of the nucleus in an external magnetic field in the $z$ direction is $U=-\mu_{z} B_{0}=-\gamma \hbar I_{z} B_{0}$ where values of $I_{z}, m_{I}$ are quantized according to $m_{I}=I, I-1, I-2, I-3, \ldots-1[75,76]$. 

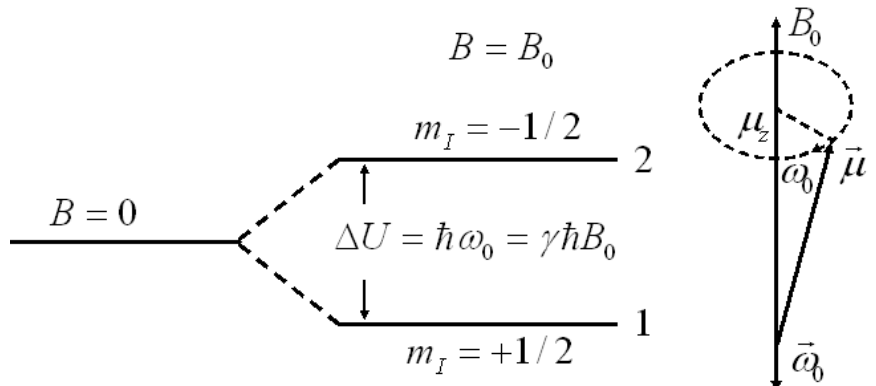

Figure 7 a) The two magnetic energy states for the spin, $I=1 / 2$ single proton of a hydrogen atom in a magnetic field. b) Time variation of the magnetic moment of the proton in magnetic field $B_{0}$ with precession frequency, $\omega_{0}=\gamma B_{0}$, the fundamental resonant frequency from a).

For most nuclear species the $z$-component of the magnetization, $M$ grows exponentially until reaching equilibrium according to $M_{z}(t)=M_{0}\left(1-e \exp -t / T_{1}\right)$ where $T_{1}$ is the spin-lattice relaxation time. Of interest for the noetic interferometer is the fact that (Fig. 9.14a) as $\mu$ precesses cyclically from $m_{I}=-1 / 2$ to $m_{I}=+1 / 2$ the nucleons experience a torque, $\tau$ changing $J$ by $\tau=d J / d t$ or $\mu \times B=d J / d t$. Under thermal equilibrium the x-y components are zero; but $M_{z}$ can be rotated into the x-y plane creating transverse $M_{x}$ and $M_{y}$ components $d M / d t=\gamma M \times B$ for the entire system by applying a rotating circularly polarized oscillating magnetic field $2 B_{1} \cos \omega t \hat{i}$ of frequency $\omega$ in addition to the constant magnetic field $B_{0} \hat{k}$. Now the total time dependent field decomposes into the two counterpropagating fields

$$
B_{1}(\cos \omega t \hat{i}+\sin \omega \hat{t j})+B_{1}(\cos \omega t \hat{i}-\sin \omega \hat{t j}) \text {. }
$$

This more complicated form for use with multiple applied fields is necessary, as described below, for use with the Sagnac Effect, quadrupole, and dipole dynamics $[77,78]$ required to operate the noetic interferometer.

Nuclear Quadrupole Resonance (NQR) is a form of NMR in which quantized energy level transitions are induced by an oscillating RF magnetic field in the electric quadrupole moment of nuclear spin systems rather than the magnetic dipole moment. The nuclear quadrupole moment, $Q$ is based on the nuclear charge distributions $\rho(r)$ departure from spherical symmetry defined as the average value of $1 / 2\left(3 z^{2}-r^{2}\right) \rho(r)$ over the nuclear volume. $Q$ has the dimension of area where the nuclear angular momentum, for which $m_{I}=I$ where $I$ is the nuclear spin quantum number and $m_{I}$ is the quantum number for the $z$ component of the spin $m_{I}=-1,+1, \ldots, I-1, I$. Nuclei with $I=0$ have no magnetic moment and are therefore magnetically inert. Similarly in order for $Q=0$ the nucleus must be spherical with spin $I \geq 0$. For spin $I=1 / 2$ nuclei have dipole moments, $\mu$ but no $Q$. $Q$ is positive for prolate nuclei and negative for oblate nuclei $[79,80]$.

For an isolated nucleus in a constant magnetic field $H_{0}$ with nuclear spin number $I>0$ the nucleus posses a magnetic moment. From Quantum Theory (QT) the length of the nuclear angular momentum vector is $\left[I\left(+_{I}\right)\right]^{1 / 2} \hbar$ where measurable components are given by $m \hbar$ with $m$ the magnetic quantum number taking any $(2 I+I)$ value from the series $I, I-I, I-2, \ldots,-(I-I),-I$. For the $I=3 / 2$ case there are four values along the direction of the applied magnetic field $H_{0}$.

Of the three types of spin-spin coupling, this experiment relies the hyperfine interaction for electron-nucleus coupling, specifically the interaction of the nuclear electric quadrupole moment induced by an applied oscillating RF electric field to act on the nuclear magnetic dipole moment $\mu$. When the electron and nuclear spins are strongly aligned along their $z$-components the Hamiltonian is $-m \cdot B$, and if $B$ is in the $z$ direction

$$
H=-\gamma_{N} I \cdot B=-\gamma_{N} B I_{x}
$$

with $m=\gamma_{N} I, \gamma_{N}$ the magnetogyric ratio $\gamma_{N}=e \hbar / 2 m_{p}$ and $m_{p}$ the mass of the proton [81].

Radio frequency excitation of the nuclear magnetic moment, $\mu$ to resonance occurs for a nucleus collectively 
which rotates $\mu$ to some angle with respect to the applied field $B_{0}$. This produces a torque $\mu_{i} \times B_{0}$ causing the angular momentum, $\mu$ itself to precess around $B_{0}$ at the Larmour frequency $\omega_{L}=\gamma_{N} B_{0}$ [81-83]. This coherent precessing of $\mu$ can also induce a 'voltage' in surrounding media, an energy component of the Hamiltonian to be utilized (Fig. 9.14) to create interference in the structure of spacetime [23].

Metaphorically this is like dropping stones in a pool of water: One stone creates concentric ripples; two stones create domains of constructive and destructive interference. Such an event is not considered possible in the standard models of particle physics, quantum theory and cosmology. However Noetic science uses extended versions of these theories wherein a new teleological action principle is utilized to develop what might be called a 'transistor of the vacuum'. Just as standard transistors and copper wires provide the basis for almost all modern electronic devices; This L.O.V.E.R. using the information content of spacetime geodesics (null lines) will become the basis of many forms of Noetic Technologies. After a bit of thought we thought a little fun was warranted and came up with a name for the core of this noetic class of vacuum technologies: Laser Oscillated Vacuum Energy Resonator (L.O.V.E.R.). Wouldn't it be a kick if for the next 1,000 years noetic or anthropic technologies are 'full of love'?

Simplistically in this context, utilizing an array of modulated tunable lasers, atomic electrons are RF pulsed with a resonant frequency that couples them to the magnetic moment of the nucleons such that a cumulative interaction is created to dramatically enhance the Haisch-Rueda inertial back-reaction [84-87]. The laser beams are counterpropagating producing a Sagnac effect Interferometry to maximize the violation of Special Relativity. This is the $1^{\text {st }}$ stage of a multi-tier experimental platform designed (according to the tenets of Noetic Field Theory) to 'open a hole' in the fabric of spacetime in order to isolate and utilize the force $\hat{F}_{U}$ of the Unitary Field.

The interferometer utilized as the basis for our vacuum engineering research platform has been dubbed the Laser Oscillated Vacuum Energy Resonator. It is a multi-tiered device. The top tier is comprised of counter-propagating Sagnac effect ring lasers that can be built into an IC array of 1,000+ ring lasers. If each microlaser in the array is designed to be counterpropagating, an interference phenomena called the Sagnac Effect occurs that violates special relativity in the small scale [88]. This array of RF modulated Sagnac-Effect ring lasers provides the top tier of the multi-tier L.O.V.E.R. Inside the ring of each laser is a cavity where quantum effects called Cavity Quantum Electrodynamics (C-QED) may occur. A specific molecule is placed inside each cavity. If the ring laser array is modulated with resonant frequency modes chosen to achieve spin-spin coupling with the molecules electrons and neutrons, by a process of Coherent Control [89] of Cumulative Interaction an inertial back-reaction is produced whereby the electrons also resonate with the spacetime backcloth in order to 'open an oscillating hole' in it. This requires a form of RQFT compatible with the 12D version of M-theory called F-Theory [24] relying on the symmetry conditions of HAM cosmology within which it is cast [29-31].

\section{LASER OSCILLATED VACUUM ENERGY RESONATOR (L.O.V.E.R.) Multi-Tiered Experimental Platform}

\begin{tabular}{l} 
TIER-I $\begin{array}{l}\text { Applied Tunable Laser RF Modulated Pulsed } \\
\text { Quadrupole Resonant Counter-Propagating } \\
\text { Sagnac Effect Interferometry of Electrons }\end{array}$ \\
\begin{tabular}{|l|}
\hline For the Purpose of Spin-Spin Coupling of Tier-I \\
Electrons to the Magnetic Moment of the Nucleons
\end{tabular} \\
TIER-III $\begin{array}{l}\text { By HD RQFT Tier- I \& II Undergo Resonant Coupling } \\
\text { with the Beat Frequency of the Fabric of Spacetime }\end{array}$ \\
TIER-IV $\begin{array}{l}\text { Producing a Multi-Tier Cumulative Interaction of } \\
\text { Tier - I - II } \| \text { II to Destructively Interfere with the } \\
\text { Annihilation \& Creation operators of Spacetime }\end{array}$ \\
\hline
\end{tabular}

\begin{tabular}{|c|c|}
\hline \multicolumn{2}{|c|}{ Hierarchical Harmonic Oscillator Parameters } \\
\hline classical & $X=A \cos (\omega t)$ \\
\hline quantum & $\frac{\hbar^{2}}{2 m} \frac{d^{2} \psi}{d x^{2}}+\left(E-\frac{k x^{2}}{2}\right) \psi=0$ \\
\hline $\begin{array}{l}\text { annihilation } \\
\text { creation }\end{array}$ & $x(t)=x_{0}\left[a \exp (-i \omega t)+a^{\dagger} \exp (i \omega t)\right]$ \\
\hline $\begin{array}{l}\text { future-past } \\
\text { retarded- } \\
\text { advanced }\end{array}$ & $\begin{array}{l}F_{1}=F_{0} e^{-i k x} e^{-2 \pi i f f}, F_{2}=F_{0} e^{i k \pi} e^{-2 \pi i f f}, \\
F_{3}=F_{0} e^{-i k x} e^{2 x i f f}, F_{4}=F_{0} e^{i k x} e^{2 \pi f f}\end{array}$ \\
\hline incursive & $\frac{d r(t+\Delta t)}{d t}-v(t)=0, \frac{d v(t+\Delta t)}{d t}+\omega^{2}=0$ \\
\hline
\end{tabular}

Figure 8 A) Design elements for the HD Cavity-QED trap of the Noetic Interferometer postulated to constructively-destructively interfere with the topology of the 12D spacetime manifold to manipulate the unitary field. Substantial putative effects are possible if cumulative interactions of the interference nodes of the cyclotron resonance hierarchy produce shock waves. B) Basic mathematical components of the applied harmonic oscillator: classical, quantum, relativistic, transactional and incursive are required in order to achieve coherent control of the cumulative resonance coupling hierarchy in order to produce harmonic nodes of destructive and constructive interference in the spacetime backcloth.

The first step in the interference hierarchy (Fig. 9.15) is to establish an inertial back-reaction between the modulated electrons and their coupled resonance modes with the nucleons. The complete nature of inertia remains a 
mystery [90]. But if one follows the Sakarov [91] and Puthoff [92] conjecture, the force of gravity and inertia, the initial resistance to motion, are actions of the vacuum zero-point field. Therefore the parameter $m$ in Newton's second law $f=m a$ is a function of the zero-point field [84-86,93-95]. Newton's third law states that 'every force has an equal and opposite reaction'. Haisch \& Rueda [84-87] claim vacuum resistance arises from this reaction force, $f$ $=-f$. We have also derived an electromagnetic interpretation of gravity and electromagnetism [96] that suggests this inertial back-reaction is like an electromotive force ${ }^{2}$ of the de Broglie matter-wave field in the spin exchange annihilation creation process inherent in a hysteresis of relativistic spacetime fabric. In fact we go further to suggest that the energy responsible for Newton's third law is a result of the continuous-state flux of the ubiquitous noetic field [99]. For the L.O.V.E.R. we assume the Haisch-Rueda postulate is correct

$$
f=\frac{d \rho}{d t}-\lim _{\Delta t \rightarrow 0} \frac{\Delta \rho}{\Delta t} \equiv \frac{d \rho_{*}}{d t_{*}}-\lim _{\Delta t_{*} \rightarrow 0} \frac{\Delta \rho_{*}}{\Delta t_{*}}=f_{*}
$$

where $\Delta \rho$ is the impulse given by the accelerating agent and $\Delta \rho_{*}^{z p}=-\Delta \rho_{*}[84-87]$.

The cyclotron resonance hierarchy must also utilize the proper beat frequency of the continuous-state dimensional reduction spin-exchange compactification process inherent in the symmetry of noetic spacetime naturally 'tuned' to make the speed of light $c \equiv c$. With this apparatus in place noetic theory suggests that destructive-constructive C-QED interference of the spacetime fabric occurs such that the noeon eternity wave, $\aleph$ of the unitary field, $U_{F}$ is harmonically (like a holophote) released into the cavity of the detector array. Parameters of the Dubois incursive oscillator are also required for aligning the interferometer hierarchy with the beat frequency of spacetime.

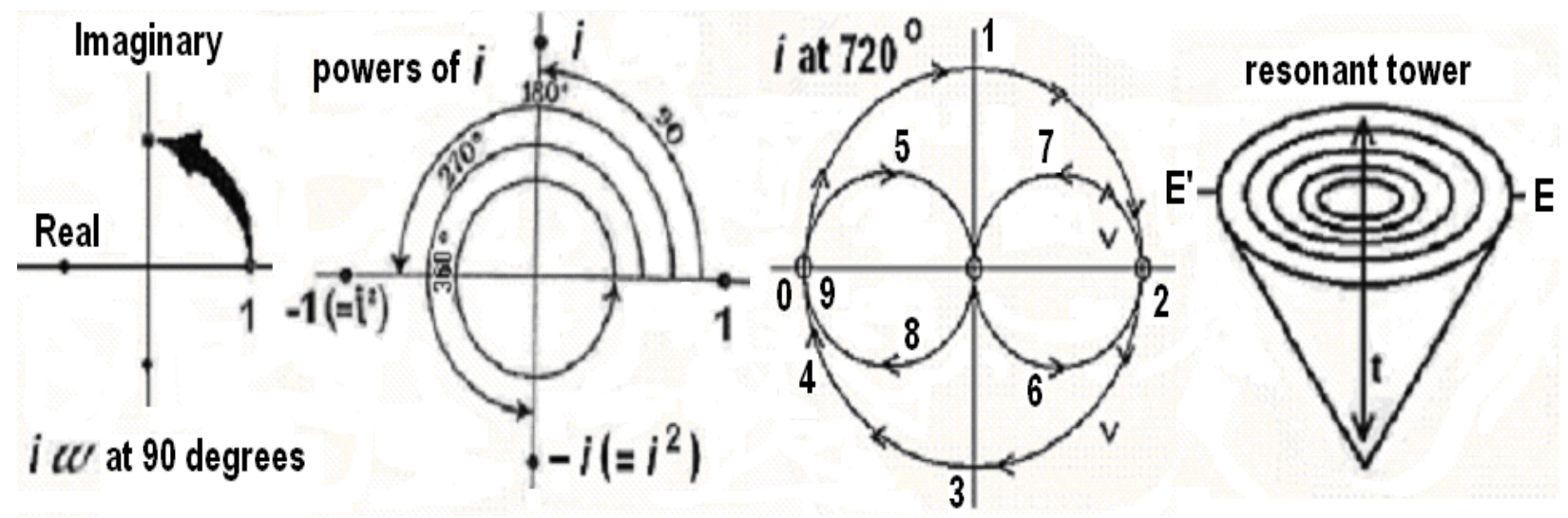

Figure 9 Powers of $i$ in the complex plane. For $90^{\circ}$ to $360^{\circ}$ the concept can be readily illustrated in $2 \mathrm{D}$; but for $720^{\circ}$ and above $4 \mathrm{D}$ is required which cannot easily be depicted in $3 \mathrm{D}$ so the representation in $9 \mathrm{c}$ ) is used, which might also be represented by a Klein bottle which was not used because the torus in 9c) more easily shows the rotation topology, which for spin $1 / 2$ is the Dirac rotation of the electron. 9d) is a simplistic representation of a powers of $i$ resonance hierarchy.

If the water wave conception for the 'Dirac sea' is correct, the continuous state compactification process contains a tower of spin states from spin 0 to spin 4 . Spin 4 represents the unified field and makes cyclic correspondence with spin 0 where Ising lattice spin flips create dimensional jumps. Spin $0,1 / 2,1, \& 2$ remain in standard form. Spin three is suggested to relate to the orthogonal properties of atomic energy levels and space quantization. Therefore the spin tower hierarchy precesses through $0,720^{\circ}, 360^{\circ}, 180^{\circ}, 90^{\circ} \& 0(\infty)$ as powers of $i$ as illustrated in Fig. 9.16

\footnotetext{
${ }^{2}$ Electromotive force, $E$ : The internal resistance $r$ generated when a load is put upon an electric current $I$ between a potential difference $V$, i.e. $r=(E-V) / I$.
} 


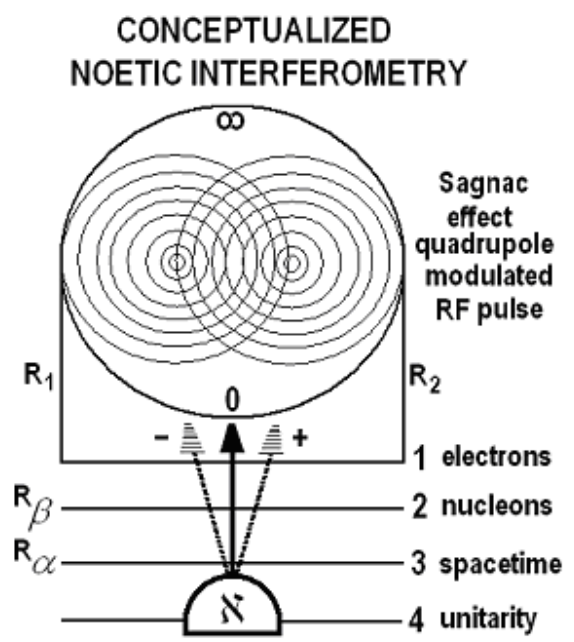

Figure 10 Conceptualized Ising model Riemann sphere cavity-QED multi-level Sagnac effect interferometer designed to 'penetrate' space-time to emit the 'eternity wave, $\aleph$ '. Experimental access to vacuum structure or for surmounting the uncertainty principle can be done by two similar methods. One is to utilize an atomic resonance hierarchy and the other a spacetime resonance hierarchy. The spheroid is a 2D representation of a HD Ising model Riemann sphere able to spin-flip from zero to infinity.

As illustrated in Fig. 9.10 the coherent control of the multi-level tier of cumulative interactions relies on full utilization of the continuous-state cycling inherent in parameters of HAM cosmology [29-31]. What putatively will allow noetic interferometry to operate is the harmonic coupling to periodic modes of Dirac spherical rotation in the symmetry of the HD geometry. The universe is no more classical than quantum as currently believed; reality rather is a continuous state cycling of nodes of classical to quantum to unitary, $C \rightarrow Q \rightarrow U$. Space does not permit detailed delineation of the parameters of HAM cosmology here; more detailed discussion can be found in [29-31]. The salient point is that cosmology, the topology of spacetime itself, has the same type of spinorial rotation and wave-particle duality Dirac postulated for the electron. Recall that the electron requires a $4 \mathrm{D}$ topology and $720^{\circ}$ for one rotation instead of the usual $360^{\circ}$ to complete a rotation in 3D. The hierarchy of noetic cosmology is cast in 12D such that the pertinent form of relativistic quantum field theory has significantly more degrees of freedom whereby the modes of resonant coupling may act on the structural-phenomenology of Dirac 'sea' itself rather than just the superficial zero-point field surface approaches to vacuum engineering common until now.

The parameters of the noetic oscillator (Figs. 9.17,9.18) may best be implemented by RQFT using a form of de Broglie fusion. According to de Broglie a spin 1 photon can be considered a fusion of a pair of spin 1/2 corpuscles linked by an electrostatic force. Initially de Broglie thought this might be an electron-positron pair and later a neutrino and antineutrino. "A more complete theory of quanta of light must introduce polarization in such a way that to each atom of light should be linked an internal state of right and left polarization represented by an axial vector with the same direction as the propagation velocity" [97]. These prospects suggest a deeper relationship in the structure of spacetime of the Cramer type [18] (Fig. 11).

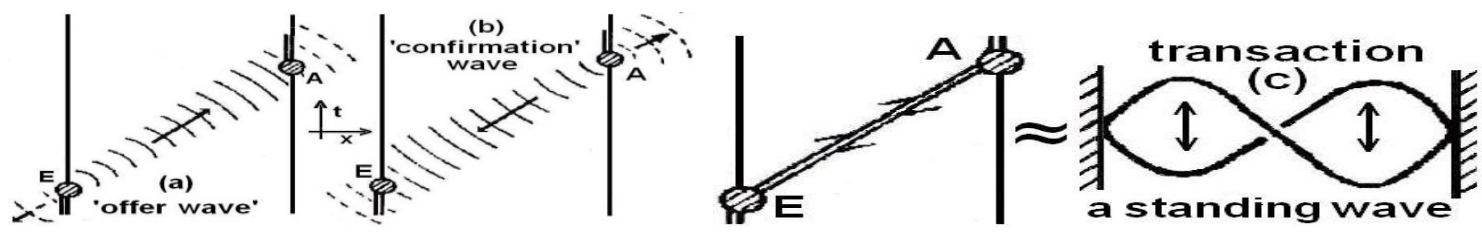

Figure 11 of an HD future-past advanced-retarded standing or stationary wave. Figs. Adapted from Cramer [18].

The epistemological implications of a 12D RQFT must be delineated. The empirical domain of the standard model relates to the 4D phenomenology of elementary particles. It is the intricate notion of what constitutes a particle that concerns us here - the objects emerging from the quantized fields defined on Minkowski spacetime. This domain for evaluating physical events is insufficient for our purposes. The problem is not only the additional degrees of freedom and the associated extra-dimensionality, or the fact that 'particles' can be annihilated and created but that in HAM cosmology they are continuously annihilated and recreated within the holograph as part of the annihilation and recreation of the fabric of spacetime itself. This property is inherent in the 12D Multiiverse because temporality is a subspace of eternity [32,33]. This is compatible with the concept of a particle as a quantized field. 
What we are suggesting parallels the wave-particle duality in the propagation of an electromagnetic wave. We postulate this as a property of all matter and spacetime albeit as continuous-state standing waves.

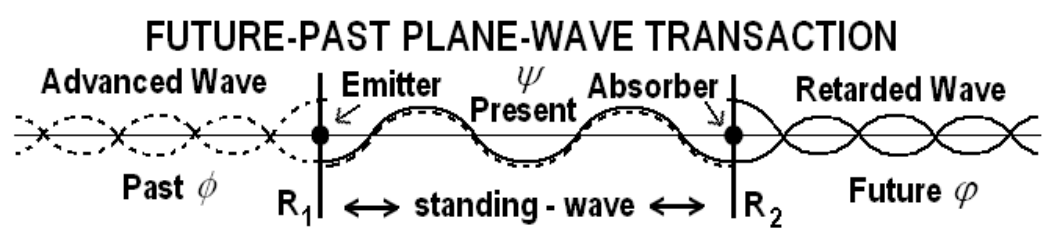

Figure 12 Structure of a transaction (present state or event) where the present is a standing-wave of future-past elements. The separation of these parameters in terms of de Broglie's fusion model is suggested to allow manipulation of the harmonic tier of the L.O.V.E.R. with respect to T-Duality or mirror symmetry.

For a basic description, following de Broglie's fusion concept, assume two sets of coordinates $x_{1}, y_{1}, z_{1}$ and $x_{2}, y_{2}, z_{2}$ which become

$$
X=\frac{x_{1}+x_{2}}{2}, \quad Y=\frac{y_{1}+y_{2}}{2}, \quad Z=\frac{z_{1}+z_{2}}{2} .
$$

Then for identical particles of mass $m$ without distinguishing coordinates, the Schrödinger equation (for the center of mass) is

$$
-i \hbar \frac{\partial \psi}{\partial t}=\frac{1}{2 M} \Delta \psi, \quad M=2 m
$$

In terms of Fig. 9.20, Eq. 9.16 corresponds to the present and Eq. 9.17a corresponds to the advanced wave and $(9.17 b)$ to the retarded wave [63].

$$
-i \hbar \frac{\partial \phi}{\partial t}=\frac{1}{2 M} \Delta \phi, \quad-i \hbar \frac{\partial \varphi}{\partial t}=\frac{1}{2 M} \Delta \varphi .
$$

Extending Rauscher's concept for a complex eight space differential line element $d S^{2}=\eta_{\mu \nu} d Z^{\mu} d Z^{* v}$, where the indices run 1 to $4, \eta_{\mu v}$ is the complex eight-space metric, $Z^{\mu}$ the complex eight-space variable and where $Z^{\mu}=X_{\mathrm{Re}}^{\mu}+i X_{\mathrm{Im}}^{\mu}$ and $Z^{* V}$ is the complex conjugate [98,99], to $12 \mathrm{D}$ continuous-state HAM spacetime; we write just the dimensions for simplicity and space constraints

$$
x_{\mathrm{Re}}, y_{\mathrm{Re}}, z_{\mathrm{Re}}, t_{\mathrm{Re}}, \pm x_{\mathrm{Im}}, \pm y_{\mathrm{Im}}, \pm z_{\mathrm{Im}}, \pm t_{\mathrm{Im}}
$$

where \pm signifies Wheeler-Feynman/Cramer type future-past/retarded-advanced dimensions. This dimensionality provides an elementary framework for applying the hierarchical harmonic oscillator parameters suggested in Figs. 9.15 and 9.18 .

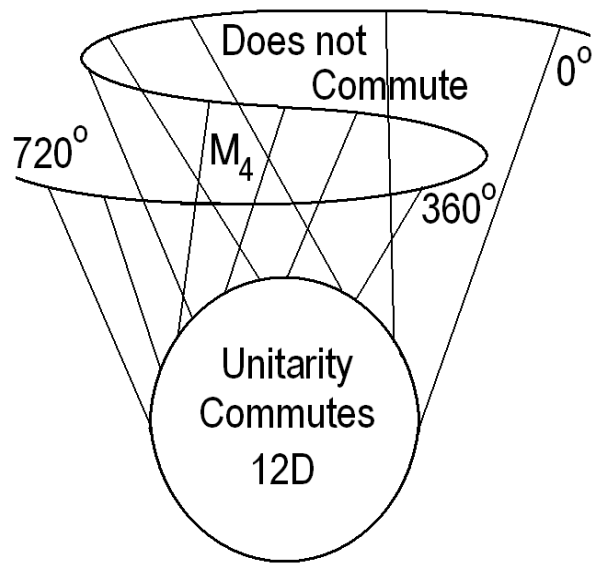

Figure 13 4D Minkowski space is like an HD quantum 'knot' tangled in a manner that the component phases do not commute. Conceptually this is like the observed retrograde motion of the planets. This is the same as A 3D view of a 4D Dirac rotation or 'pinch' of the $360-720^{\circ}$ spinor rotation of the electron. 


\section{CONCLUSIONS}

If the Noetic Interferometer is able to isolate and manipulate the eternity wave, $\aleph$ it will become a primary research platform for developing a whole new class of vacuum based technologies; whereas one could say virtually all electronic devices up to now are based on transistors and copper wires. The L.O.V.E.R. could be called a transistor of the vacuum, where rather than copper wires, the geodesics or null lines of spacetime are utilized to transfer information topologically with no exchange particle mediating the 'interaction' which perhaps should be called a correlation or entanglement in this scenario to distinguish phenomenology versus ontology.

This brief introduction is only a primitive overview of introducing the anticipated new field of $\aleph$-wave (eternity-wave) vacuum engineering that as Cramer stated in the $1^{\text {st }}$ sentence of this chapter will revolutionize many fields of science [100].

When the great innovation appears, it will most certainly be in a muddled, incomplete form. To the discoverer himself it will be only half-understood; to everyone else it will be a mystery. For any speculation which does not at first glance look crazy, there is no hope [101].

Finally we stress that vacuum energy is not 'produced' by the noetic interferometer. The interferometer manipulates the boundary conditions 'insulating' or 'hiding' the unitary geodesics of spacetime by constructive and destructive interference allowing the holophotic release of unitary noeons by completing a cascading water-wheel like circuit already existing behind the usual spacetime domain walls of reality. Probably L.O.V.E.R. vacuum energy is emitted into the L.O.V.E.R. as a form of superradiance [102] of the hysteresis loop of least-unit parallel transport.

We have found already that a fair number of our colleagues want to dismiss this model right off because of its utilization of XD. This is the sort of myopic view that has consistently plagued the history of science whenever 'bigleap' innovation occurs. We hope readers here will not fall into this quagmire! The model is empirically testable which hopefully makes up for some of the lack of precision in our axiomatic approach or thin rigor in portions of our attempts at formalism. In addition to the protocols presented here we have described already an experiment to utilize the noetic $\aleph$-wave for the putative manipulation of prion protein conformation [23].

\section{REFERENCES}

1 Elitzur, A. C. \& Vaidman, L. (1993) Quantum mechanical interaction-free measurements. Found. Phys. 23; $987-997$.

2 Kwiat, P., Weinfurter, H., Herzog, T., Zeilinger, A. \& Kasevich, M. (1995) Interaction-free quantum measurements. Phys. Rev. Lett. 74, 4763-4766.

3 du Marchie Van Voorthuysen, E.H. (1996). Realization of an interaction-free measurement of the presence of an object in a light beam, Am. J. Phys. 64:12; 1504-1507; or arXiv:quant-ph/9803060 v2 26.

4 Helmer, F., Mariantoni, M., Solano, E. \& Marquardt, F. (2008) Quantum, Zeno effect in the quantum non-demolition detection of itinerant photons, arXiv:0712.1908v2.

5 Facchi, P. Lidar, D.A. \& Pascazio, S. (2004) Unification of dynamical decoupling and the quantum Zeno effect, Phys Rev A 69, 032314; or arxiv:quant-ph/0303132.

6 Sudarshan, E.C.G. \& Misra, B. (1977) The Zeno's paradox in quantum theory, J Mathematical Physics 18:4; 756-763.

7 Facchi, P. \& Pascazio, S. (2002) Quantum Zeno subspaces, arXiv:quant-ph/0201115v2.

8. Sudarshan, E.C.G.; Misra, B. (1977). "The Zeno's paradox in quantum theory". Journal of Mathematical Physics 18 (4): $756-$ 763. doi:10.1063/1.523304

9. T. Nakanishi, K. Yamane, and M. Kitano: Absorption-free optical control of spin systems: the quantum Zeno effect in optical pumping Phys. Rev. A 65, 013404 (2001)

10. P. Facchi, D. A. Lidar, \& S. Pascazio Unification of dynamical decoupling and the quantum Zeno effect Physical Review A 69, $032314(2004)$

11. A. Degasperis, L. Fonda \& G.C. Ghirardi (1974). "Does the lifetime of an unstable system depend on the measuring apparatus?". Il Nuovo Cimento A 21 (3): 471-484. doi:10.1007/BF02731351

^ Christof Teuscher \& Douglas Hofstadter (2004). Alan Turing: Life and Legacy of a Great Thinker. Springer. p. 54. ISBN 3540200207.

12. John von Neumann (1932). Mathematische Grundlagen der Quantenmechanik. Springer. Chapter V.2. ISBN 3540592075. (See also: J. von Neumann (1955). Mathematical Foundations of Quantum Mechanics. Princeton: Princeton University Press. p. 366. ISBN 0691028931.); Michael B. Mensky (2000). Quantum Measurements and Decoherence. Springer. p. §4.1.1, pp. 315 ff. ISBN 0792362276.; Ch. Wunderlich \& Ch. Balzer (Benjamin Bederson \& H Walther, editors) (2003). Advances in Atomic, Molecular, and Optical Physics: Vol. 49. Academic Press. ISBN 0120038498. 
13 Gerlach, W \& Stern, O. (1922) Das magnetische moment des silberatoms, Zeitschrift für Physik 9, 353-355.

14 Young, T. (1804) Experiments and calculations relative to physical optics, Philosophical Trans. of the Royal Society of London 94, 1-16.

15 Kwiat, P.G., Weinfurter, H., Herzog, T., Zeilinger, A. \& Kasevich, M. (1995) Experimental realization of 'interaction-free' measurements,

in D.M. Greenberger \& A. Zelinger (eds.) Fundamental Problems in Quantum Theory, A Conference held in Honor of Professor John A. Wheeler, Annals of the New York Academy of Science, Vol. 755, p. 383, New York: New York Academy of Science.

16 Jaekel, M.T. \& S. Reynaud, S. (1990) Quantum limits in interferometric measurements, Europhys. Lett. 13, 301.

17 Cramer, J.G. (2006) A transactional analysis of interaction-free measurements, Foundations of Physics Letters 19: 1; 63-73; or arXiv:quant-ph/0508102v23, 2008.

18 Everett, H. (1957) Relative state formulation of quantum mechanics, Reviews of Modern Physics, Vol 29, pp 454-462.

19 Bohm, D. \& Vigier, J-P (1954) Model of the causal interpretation of quantum theory in terms of a fluid with irregular

fluctuations, Phys. Rev. 96:1; 208-217.

$20 \mathrm{Kaku}$, M. (1999) Introduction to Superstrings and M-Theory, New York: Springer.

21 Dirac, P.A.M. (1952) Is there an ether? Nature, 169: 172.

22 Pettroni, N.C. \& Vigier, J-P (1983) Dirac's aether in relativistic quantum mechanics, Foundations Physics, 13:2, 253-285.

23 Vigier, J-P (1980) De Broglie Waves on Dirac Aether: A Testable Experimental Assumption, Lettere. al Nuovo Cimento, 29; 467-475.

24 Lehnert, B. (2002) New developments in electromagnetic field theory, in R.L. Amoroso, G. Hunter, M. Kafatos \& J-P Vigier (eds.) Gravitation \& Cosmology: From the Hubble Radius to the Planck Scale, Dordrecht: Kluwer Academic.

25 Lehnert, B. (1998) Electromagnetic theory with space-charges in vacuo, in G. Hunter, S. Jeffers \& J-P Vigier (eds.) Causality and Locality in Modern Physics, Dordrecht: Kluwer Academic.

26 Proca, A, (1936) Compt. Rend., 202, 1420.

27 Kotigua, R.P. \& Toffoli, T. (1998) Potential for computing in micromagnetics via topological conservation laws, Physica D, 120:1-2, pp. 139-161.

28 Witten, E. (1993) Quantum background independence In string theory, arXiv:hep-th/9306122v1

29 Sen, A. \& Zwiebach, B. (1994) Local background independence of classical closed string field theory, Nucl. Phys. B414, 649.

30 Sen, A. \& Zwiebach, B. (1994) Quantum background independence of closed string field theory, Nucl. Phys. B423, 580.

31 Cramer, J. (1986) The Transactional Interpretation of Quantum Mechanics, Rev. Mod. Phys 58, 647-687.

32 Wheeler, J.A., \& Feynman, R. (1945) Rev. Mod. Physics, 17, 157;

54 Chu, S-Y (1993) Physical Rev. L., 71, 2847.

33 Amoroso, R.L. \& Pribram, K.H. (2009) The Complementarity of Mind and Body: Realizing the Dream of Descartes, Einstein and Eccles, Cambridge: MIT University Press.

34 Amoroso, R.L. (2000) The parameters of temporal correspondence in a continuous state conscious universe, in R. Buccheri \& M. Saniga (eds.) Studies in the Structure of Time: From Physics to Psycho(patho)logy, Dordrecht Kluwer Academic.

35 Chu, M-Y.J. \& Amoroso, R.L. (2008) Empirical mediation of the primary mechanism initiating protein conformation in prion propagation, in D. Dubois (ed.) Partial Proceedings of CASYS07, IJCAS, Vol. 22, Univ. Liege Belgium.

36 Holland, P.R. (1995) The Quantum Theory of Motion: An Account of the de Broglie-Bohm Causal Interpretation of Quantum Mechanics, Cambridge: Cambridge Univ. Press.

37 Randall, L. (2005) Warped Passages, Unraveling the Mysteries of the Universe's Hidden Dimensions, New York: HarperCollins.

38 Vigier, J-P (2000) selected papers, in S. Jeffers, B. Lehnert, N. Abramson, \& L. Chebotarev (eds.) Jean-Pierre Vigier and the Stochastic Interpretation of Quantum Mechanics, Montreal: Aperion.

39 Amoroso, R.L. (2002) Developing the cosmology of a continuous state universe, in R.L. Amoroso, G. Hunter, M. Kafatos \& J-P Vigier (eds.) Gravitation \& Cosmology: From the Hubble Radius to the Planck Scale, Dordrecht: Kluwer Academic. 40 Amoroso, R.L. (2005) Paradigm for a continuous-state holographic conscious multiverse, in R.L. Amoroso \& B. Lehnert (eds.) Extending the Standard Model: Searching for Unity in Physics, Oakl.: Noetic Press.

41 Amoroso, R.L. (2009) Defining a context for the cosmology of awareness, in R.L. Amoroso \& K.H. Pribram (eds.) The Complementarity of Mind and Body: Realizing the Dream of Descartes, Einstein and Eccles, Cambridge: MIT University Press. 42 Amoroso, R.L. (2004) The fundamental limit and origin of complexity in biological systems: A new model for the origin of life, in D.M. Dubois (ed.) Computing Anticipatory Systems, AIP Conf. Proceedings Vol. 718, pp. 144-159, Melville: American Inst. of Physics.

43 Amoroso, R.L. (2009) The physical origin of the principle of self-organization driving living systems, in R.L. Amoroso \& K.H. Pribram (eds.) The Complementarity of Mind and Body: Realizing the Dream of Descartes, Einstein and Eccles, Cambridge: MIT Univ. Press.

44 Kafatos, M. Roy, S. \& Amoroso, R. (2000) Scaling in Cosmology \& the Arrow of Time, in Buccheri, di Gesu \& Saniga, (eds.) Studies on Time, Dordrecht: Kluwer Academic.

45 Amoroso, R.L. (1996) The production of Fröhlich and Bose-Einstein coherent states in in vitro paracrystaline oligomers using phase control laser interferometry, Bioelectrochemistry \& Bioenergetics, 41:1, pp.39-42.

46 Fröhlich, H. (1968) Long-range coherence and energy storage in biological systems, Int. J. Quantum Chem. 2:641-649.

47 Preparata, G. (1995) QED Coherence in Matter, Singapore: World Scientific.

48 Driesch, H. (1914) C.K. Ogden (trans.) The History and Theory of Vitalism, London: Macmillan.

49 Amoroso, R.L. (2009) Consciousness: The philosophical foundations of noetic field theory, in R.L. Amoroso \& K.H. Pribram (eds.) The Complementarity of Mind and Body: Realizing the Dream of Descartes, Einstein and Eccles, Cambridge: MIT Univ. Press. 
50 Amoroso, R.L. \& Martin, B. (1995) Modeling the Heisenberg matrix: Quantum coherence \& thought at the holoscape matrix and deeper complementarity, in J. King \& K. H. Pribram (eds.) Scale in Conscious Experience, Mahwah: Lawrence Earlbaum. 51. Amoroso, R.L. (1997) Consciousness a radical definition: The hard problem made easy, The Noetic Journal 1:1, pp. 19-27. 52. Amoroso, R. L. (1997) A brief introduction to noetic field theory: The quantization of mind, in L. Rakic, G. Kostopoulos, D. Rakovic, \& D. Koruga (eds.) Brain and Consciousness, Belgrade: ECPD.

53. Amoroso, R.L, (2000) Consciousness, a radical definition: Substance dualism solves the hard problem, in Amoroso, R.L., Antunes, R, Coelho, C., Farias, M., Leite, A., \& Soares, P. (eds.) Science \& the Primacy of Consciousness, Orinda: Noetic Press. 54 Amoroso, R.L (1999) An introduction to noetic field theory: The quantization of mind, Noetic J. 2:1, pp. 28-37.

55. Amoroso, R.L. \& Martin, B.E. (2002) Consciousness: 'A thousand points of light', The emergence of self-organization from the noumenon of the conscious universe, The Noetic Journal, 3:4, 289-311.

56 Amoroso R.L. (2003) Awareness: physical cosmology of the fundamental least unit, Noetic Journal 4:1, 1-15

57 Amoroso, R.L. (2003) The Fundamental Limit and Origin of Biological Systems, Noetic Journal 4:1; 24-32.

58. Amoroso, R.L. (2003) The physical basis of qualia: Overcoming the $1^{\text {st }}$ person $3^{\text {rd }}$ person barrier, Noetic J. 4:3, pp. 212-230.

59. Amoroso, R.L. (2002) The physical basis of consciousness: A fundamental formalism, Part 1 Noesis, XXVI, Bucharest:

Romanian Acad.

60. Dubois, D.M. (2001) Theory of incursive synchronization and application to the anticipation of delayed linear and nonlinear systems, in D.M. Dubois (ed.) Computing Anticipatory Systems: CASYS 2001, 5th Intl Conf., Am Inst of Physics: AIP Conf. Proceedings 627, pp. 182-195.

61. Antippa, A.F. \& Dubois, D.M. (2008) The synchronous hyperincursive discrete harmonic oscillator, in D. Dubois (ed.) proceedings of CASYS07, preprint.

62. Dubois, D.M. (2008) The quantum potential and pulsating wave packet in the harmonic oscillator, in D. Dubois (ed.) proceedings of CASYS07, preprint.

63. Antippa, A.F. \& Dubois, D.M. (2004) Anticipation, orbital stability and energy conservation in discrete harmonic oscillators, in D.M. Dubois (ed.) Computing Anticipatory Systems, AIP Conf. Proceedings Vol. 718, pp.3-44, Melville: American Inst. of Physics.

64. Icke, V. (1995) The Force of Symmetry, Cambridge: Cambridge Univ. Press.

65. Mackay, A.L. \& Pawley, G.S. (1963) Bravais Lattices in Four-dimensional Space, Acta. cryst. 16: 11-19.

66. Calzolari, A., Marzari, N., Souza, I. \& Nardelli, M.B. (2003) arXiv:cond-mat/0311018.

67. Schwinger, J. (1992) Casimir energy for dielectrics, Proc. Nat. Acad. Sci. USA 89, 4091-3.

68. Schwinger, J. (1993) Casimir light: The source, Proc. Nat. Acad. Sci USA 90, 2105-6.

69. Schwinger, J. (1994) Casimir energy for dielectrics: spherical geometry, Proc. Nat. Acad. Math. Psych. 41:64-67, San

Ffrancisco: W.H. Freeman.

70. Witten, E. (1996) Reflections on the fate of spacetime, Phys. Today, (April) pp. 24-30.

71. Amoroso, R.L. (2005) Application of double-cusp catastrophe theory to the physical evolution of qualia: Implications for paradigm shift in medicine \& psychology, in G.E. Lasker \& D.M. Dubois (eds) Anticipative \& Predictive Models in Systems Science, Vol. 1, pp. 19-26, Windsor: The International Institute for Advanced Studies in Systems Research \& Cybernetics. 72. Gilmore, R. (1981) Catastrophe Theory for Scientists \& Engineers, New York: Dover.

73. Poston T. \& Stewart, I (1978) Catastrophe Theory \& Its Applications, New York: Dover; Gilmore, R. (1981) Catastrophe Theory for Scientists \& Engineers, Dover.

74. Qin, S. et al. (2001) Int. J of Solids \& Structures, 38, pp. 8093-8109.

75. Stevens, H.H. (1989) Size of a least unit, in M. Kafatos (ed.) Bell's Theorem, Quantum Theory and Conceptions of the Universe, Dordrecht: Kluwer Academic.

76. Cardone, F. \& Mignani, R. (2004) Energy and Geometry - An Introduction to Deformed Special Relativity, Singapore: World Scientific.

77. Cardone, F., Marrani, A. \& Mignani, R. (2003) The electron mass from deformed special relativity, Electromagnetic Phenomena Vol.3, N.1; 9, special number dedicated to Dirac's centenary; (2008) hep-th/0505134.

78. Cardone, F., Marrani, A. \& Mignani, R. (2008) A new pseudo-Kaluza-Klein scheme for geometrical description of interactions, arXiv:hep-th/0505149v1.

79. Bohm, D. (1963) Quantum Theory, pg. 353, Englewood Cliffs: Prentice-Hall.

80. Messiah, A. (1999) Quantum Mechanics, pg 438, 444, New York: Dover.

81. Hatfield, B. (1992) Quantum Field Theory of Point Particles and Strings, Reading: Addison-Wesley.

82. Grotz, K. \& Klapdor, H.V. (1990) The Weak Interaction in Nuclear, Particle and Astrophysics, New York: Adam Hilger.

83. Slichter, C.P. (1990) Principles of Magnetic Resonance, $3^{\text {rd }}$ edition, Springer Series in Solid-State Sciences 1, New York: Springer.

84. Schumacher, R.T. (1970) Introduction to Magnetic Resonance, Menlo Park: Benjamin-Cummings.

85. Farrar, T.C. \& Becker, E.D. (1971) Pulsed and Fourier Transform NMR, New York: Academic Press.

86. Abragam, A. (1961) Principles of Nuclear Magnetism, Oxford: Clarendon Press.

87. Dehmelt, H.G. (1954) Nuclear quadrupole resonance, Am. J. Physics, 22:110.

88. Semin, G.K, Babushkina, T.A. \& Yakobson, G.G. (1975) Nuclear Quadrupole Resonance in Chemistry, New York: Wiley. 89. Atkinson, P.W. (1994) Molecular Quantum Mechanics, $2^{\text {nd }}$ edition, Oxford: Oxford University Press.

90. Hausser, O. (1974) Coulomb reorientation, in J. Cerny (ed.) Nuclear Spectroscopy and Reactions, Part C, New York: Academic Press.

91. Humieres, D., Beasley, M.R., Huberman, B.A. \& Libchaber, A. (1982) Chaotic states and routes to chaos in the forced pendulum, Physical Rev A, 26:6, 3483-34.

92. Rueda, A. \& Haisch, B. (1998) Contributions to inertial mass by reaction of the vacuum to accelerated motion, Found. of Phys, 28:7, 1057-1108. 
93. Rueda, A. \& Haisch, B. (1998) Physics Lett. A, 240, 115.

94. Rueda, A., Haisch, B.\& Puthoff, H.E. (1994) Phys.Rev. A, 49, 678.

95. Rueda, A. \& Haisch, B. (2002) The inertia reaction force and its vacuum origin, in R.L. Amoroso, G. Hunter, M. Kafatos \& J-P Vigier (eds.), Gravitation \& Cosmology: From the Hubble Radius to the Planck Scale, pp. 447-458, Dordrecht: Kluwer Academic.

96. Vigier, J-P (1997) New non-zero photon mass interpretation of the Sagnac effect as direct experimental justification of the Langevin paradox, Physics Let. A, 234:2, 75-85.

97. Garcia-Ripoli, J.J., Zoller, P. \& Cirac, J.I. (2005) Coherent control of trapped ions using off-resonant lasers, Phys. Rev. A 71, $062309 ; 1-13$.

98. Vigier-J-P (1995) Derivation of inertial forces from the Einstein-de Broglie-Bohm causal stochastic interpretation of quantum mechanics, Found. Phys. 25:10, 1461-1494.

99. Sakharov, A.D. (1968) Sov. Phys. Dokl. 12, 1040.

100. Puthoff, H.E. (2002) Polarizable vacuum approach to General Relativity, in R.L. Amoroso, G. Hunter, M. Kafatos \& J-P Vigier (eds.), Gravitation \& Cosmology: From the Hubble Radius to the Planck Scale, pp. 431-446, Dordrecht: Kluwer Academic.

101. Burns, J.E. (1998) Entropy and vacuum radiation, Found. Phys. 28 (7), 1191-1207.

102. Burns, J.E. (2002), Vacuum radiation, entropy and the arrow of time, in R.L. Amoroso, G. Hunter, S. Jeffers \& M. Kafatos, (eds.) Gravitation \& Cosmology: From the Hubble Radius to the Planck Scale, Dordrecht: Kluwer Academic. 